\title{
Stakeholders' Perspectives on Rationing COVID-19 Vaccines Amidst Extreme Scarcity: A Qualitative Study
}

John Barugahare

Makerere University

Betty Kwagala

Makerere University

Joseph Ochieng ( $\square$ ochiengjoe@yahoo.com )

Makerere University

\section{Research Article}

Keywords: COVID-19 Vaccine, health resource allocation, priority setting, stakeholders' perspectives, Uganda

Posted Date: September 22nd, 2021

DOI: https://doi.org/10.21203/rs.3.rs-888025/v1

License: (c) (i) This work is licensed under a Creative Commons Attribution 4.0 International License. Read Full License 


\section{Abstract}

Background: In the context of scarce health-resources robust priority setting for access to existing interventions is critical for at least two reasons: ensuring efficient use of resources, and equitable access to existing interventions. This need is more urgent, and its achievement more intractable in severely resource-constrained health systems, of Low and Middle Income Countries, such as Uganda. COVID-19 pandemic and the resulting need for large-scale and immediate vaccination amidst extreme scarcity of vaccines in Uganda necessitated an exploration of stakeholders' perspectives on the country's priority setting for access to COVID-19 vaccines. We aimed at exploring stakeholders' perspectives on priority setting for COVID-19 vaccination in Ugandan.

Methods: We conducted key informant and in-depth interviews with key stakeholders in the COVID-19 intervention including public health experts, clinicians, policy makers, human rights experts, bioethicists, legal fraternity, biomedical scientists and members from the general public. Stakeholders' views were sought on the status of official guidelines for rationing COVID-19 vaccines; who they thought should get priority for vaccination and why; and what the process of setting such priorities should involve. Data were transcribed and analysed thematically using NVivo software (QSR international 2020).

Results: There was concern about lack of clarity regarding context-specific guidance on priorities COVID19 vaccination. This concern was corroborated by the fact that national guidelines for COVID-19 vaccination are still in draft form and inaccessible to the public. Regarding who should get priority and why, dominant views indicated more concern for efficiency-cum-effectiveness at controlling the spread of the virus, although further probing revealed that some of these views were partly motivated by equity concerns. Most respondents felt that the process of developing the needed guidelines should have employed a bottom-up approach involving rigorous community engagement.

Conclusion: Given extreme of scarce health resources, in order to strike favourable balance between effectiveness and equity in rationing COVID-19 vaccines, there is need for clear and context-specific official guidelines. To achieve such a goal, the guidelines need to be developed through a bottom-up approach involving robust public engagement.

\section{Introduction}

By the end of August 2021, the cumulative number of COVID-19 cases reported globally was about 216 million while the cumulative number of deaths was close to 4.5 million [1]. Around the same time the same figures, respectively, were over 5.6 million, and over 136,000 for the African Region [2], while in Uganda these numbers were about 112,000 and just above 3,000. In the absence of a known cure for COVID-19, one of the most feasible remedy is prevention through vaccination. However, right from the early days of the pandemic, primary focus was on developing the needed vaccine and, more important, equitable access to it between within countries [3-10]. This concern was partly motivated by the anticipation of 'vaccine nationalism' [11-19], and limited financial affordability of these vaccines by Low 
and Middle Income Countries (LMICs). A combination of these two factors would significantly shrink the amounts of vaccine dozes available to LMICs. We take it as axiomatic that extreme scarcity of vaccines would deepen challenges in the rationing of these vaccines to a point that it would be impossible to cover even those few groups of people that were recommended by the World Health Organization (WHO) to receive priority.

In view of these challenges, we focussed our study on the Ugandan context. Whereas Uganda's estimated need for COVID-19 vaccines is more than 21.9 million individuals [20] which constitute almost Forty-four million dozes (two dozes for each individual), by the beginning of September 2021, the country had received merely 2.3 million dozes, constituting about $5.2 \%$ of the needed vaccine dozes [21]. Further, even though the country intended to prioritise the categories of people as per the WHO guidance [22], the initial vaccines which were received (864,000 dozes) were slightly less than one-tenth of the dozes needed to cover two jabs for each of the 4.38 million individuals listed as priority-one groups [20].

In anticipation of the COVID-19 vaccine rationing challenges such as those being faced in Uganda, the WHO issued general guidance on how the available COVID-19 vaccines should be rationed within countries [22]. However, it was not clear whether such generalized guidance would neatly suit all countries given a lot of variability of factors across countries, which might affect equity and efficiency for example, demographic, epidemiological, economic, social factors, among others. Given this background it became important to explore perspectives of stakeholder in these countries on rationing COVID-19 vaccine, with a view of generating what might facilitate the achievement of optimal outcomes from the available resources without losing sight of equitable access to COVID-19 vaccines in those countries.

It is critical to note that generally, in the context of scarce health-resources, robust priority setting for access to the available interventions is critical for at least two major reasons: 1) the need to ensure efficiency-cum-effectiveness in the use of the available resources, and 2) ensuring equitable access to existing interventions [13, 23-32]. These two goals are more urgent, and their achievement more intractable in severely resource-constrained health systems, especially in LMICs, typical of which is Uganda. Consequently, we contend that the perspectives explored in this study, though not statistically representative of all LIMCs, have direct relevance to all countries which have found themselves with similar challenges in accessing COVID-19 vaccines.

\section{Methods}

This was a qualitative study using semi-structured interview schedules for In-depth interviews and key informant interviews. We explored stakeholders' perspectives on priority setting for COVID-19 vaccine access in Ugandan. The study involved 20 stakeholders, purposively selected based on the nature of their involvement in decisions making on health resource allocation as well as some members of the general public. Participants included three (3) bioethicists, six (6) public health experts, three (3) civil society activists, three (3) lawyers, four (4) clinicians, three (3) policy makers, four (4) members of the general 
public and eight (8) scientists. Stakeholders' views were sought on the availability of official clear guidelines for rationing COVID-19 vaccines; who should get priority for vaccination and why; and what the process of setting such priorities should be like.

Data were collected in English and involved face to face and online interviews using Zoom and telephone calls. The face to face interviews were conducted in accordance with COVID-19 prevention SOPs, including hand sanitization, physical distancing and wearing of face masks. Interviews were held in the privacy of the office of the respondent or locations they suggested as convenient and private. Sample size limit was determine by data saturation which occurred after 18 interviews. Data were transcribed verbatim and analysed through content analysis along the main themes of the study. Content analysis was conducted using a comprehensive thematic matrix, to identify themes arising from the narratives. NVivo software (QSR international 2020) was used to support data analysis and illustrative quotes were extracted.

Ethical review and approval was appropriately sought from the Makerere University School of Health Sciences Research and Ethics Committee SHSREC: 2020-043, followed by clearance by the Uganda National Council for Science and Technology HS1101ES. Only adult individuals of 18 years and above both males and females who provided written informed consent participated in the study. No participant identifying information was recorded.

\section{Results}

Whereas our study findings largely support priorities for vaccination as prescribed by the WHO and the COVAX facility, views identified in this study strongly suggest that this guidance should have been localized to ensure that it is more responsive to the local dynamics of the population. Content analysis of the collected data identified six themes including 1) participation and experience in health resource allocation, 2) Availability and awareness of guidelines for COVID-19 vaccine access, 3) Attitude regarding the need to set priorities /guidelines, 4) Considerations for priority setting regarding COVID-19 vaccination, 5) The process of Priority setting for access to COVID-19 vaccine, and 6) Challenges in priority setting for COVID-19 vaccine. In this paper we report about stakeholders' perspectives on: the availability of clear and officially recognized guidelines for rationing COVID-19 vaccines, who should get priority for vaccination and why, and what the process of setting such priorities should involve.

\section{Availability and awareness of guidelines for COVID-19 vaccine access}

On the issue of whether there was clear and context- specific national guidelines for rationing COVID-19 vaccines, the study established that at present there are no yet any approved official context-specific guidelines for rationing COVID-19 vaccine. However, the government has made efforts to draft such guidelines, which, at the time of reporting these findings, those guidelines were still in a draft form. Further, the study established that the current priorities in vaccination against COVID-19 are not entirely arbitrary. The government devised what was described, especially by respondents in the category of 
policy makers as generic 'vaccine deployment plan', based on the general guidance from the WHO and the COVAX facility. One respondent in the role policy maker revealed that:

"We have a national deployment plan- a draft and what we envisage we would do in line with the current WHO guidelines. [... ] So, we have a deployment plan draft that is generic and aligned to WHO guidelines with the COVAX facility and also, we have the interim recommendations from the NITAG that were used to form a prioritization framework to guide the program at the Ministry if the vaccine came. [...] It keeps changing and changing and why I am calling it draft it is still being reviewed by partners." IDI 001 female//policy

\section{Awareness of the existence and content of the guidelines}

Apart from those who were in the role of policy makers, a few other respondents indicated that they were aware of the existence of some form of guidance. They indicated, for example, that from what they had heard, the guidelines give priority to those who are at higher risk of being seriously affected by COVID-19, such as the elderly, people with underlying conditions, the frontline workers among others. They were also aware that the local guidelines were the same as those issued by the world Health Organization. However, the respondents who claimed that the guidelines existed, what they meant was the vaccine deployment plan as opposed to final substantive national guidelines which are currently in a draft form.

"Yes, there is strategic document for COVID-19 management particularly and all these other pillars have guidelines developed by themselves within the various pillars which gave the direction in which the response should take" IDI 002 male// clinician

"So I think guidelines were developed after the WHO recommendation of sorts and also that was based on scientific evidence that most of the cases that were getting COVID were those that were either having underlying causes or were of advanced age." IDI 012 male// scientist

"Yeah, we have heard the health workers will receive first then the teachers and who else. Those people they mentioned (I: who mentioned them?) I have heard about it, I don't know if it is real but that's what I think. [...] That's what we have heard. [...] There is a guideline, is it not true? You are doing hmm" $<$ Files\\Community \\IDI 014 male// public

However, it also emerged that many respondents were not aware of any guidelines except for what they had been reading in the press statements by the Health Minister and other government officials. They were not aware of any process that could have been followed, and what evidence was used to come up with such guidelines, especially decisions on what population groups deserve priority for COVID-19 vaccination.

"I haven't seen anything like that, but I have been seeing the Minister for Health, the permanent secretary releasing press statements." IDI 007 female//lawyer/civil society

"Yes, I don't know because I have never heard of such guidelines." IDI 013 female// public 
"I didn't know much about the process but I came across the power point presentation where somebody was planning to propose how to roll out this vaccine and priority kind of groups." IDI 002 male// clinician

On the other hand, however, some respondents expressed their uncertainty on the availability of any guidelines for access to COVID-19 vaccine. They only heard from the media about some guidance of how to roll out the COVID 19 vaccination exercise, but they could not guarantee that there were official guidelines.

"The only thing I have only been aware of is again what has come through the media is that they are going to first take care of the health workers, then the armed forces, and the security forces, and then the teachers, and I think after that they will then go to the general population." IDI 011 male// scientist

"What I have heard on radios is that, they are saying the teachers should be given the priority but I don't know how they came up with that conclusion that the teachers should be given a priority." IDI 015 female//public

To emphasize lack of clarity on the existence of the specific guidelines for access to COVID-19 vaccines, a policy maker revealed that:

"I don't know. I have not read them because the time they had was short because what they gave was an interim recommendation which may be this study will modify their recommendation." IDI 001 female// policy

Some respondents recommended the need for clear guidance and to ensure equitable allocation of the available health resources.

"I think the best thing is to put in place laws, regulations and guidelines that require practice of equity and ensuring penalties for those who have not done so, and the penalties should be in such a way that they are deterrent, so that somebody else, when they are in the position they would be scared to attempt, I think that is the best way in my view." IDI 012 male// scientist

\section{Considerations for priority setting regarding COVID-19 vaccination}

Data obtained on who should get priority in accessing the COVID-19 vaccine included both descriptive as well as normative responses. Some respondents indicated which categories of people they heard were to get priority, while some gave opinions about which categories deserve such priority. The respondents went ahead to give the reasons why they think certain categories of people should get priority.

Most of the respondents suggested that health workers should be among those to receive priority. This was due to the observation that health care workers are highly exposed to infection as they handle infected patients, and already they seemed to be overrepresented in cases of COVID-19 infections. Some respondents reasoned that a health worker who is infected is very likely to infect others, especially the patients, in the course of interacting with them, and because of the essential nature of their service which 
would be missed if they got afflicted so much by the disease. The overarching reason that was given for prioritizing health workers and other social workers was more of a pragmatic reason 'to stabilize the health system so that other health functions can go on' and ensure minimal disruption in provision of basic services including education, and the economy at large.

"I think we should set priorities using the following criteria's one, we should ask ourselves what is the critical role of these persons to the response because COVID is a public health disaster. In the ethics of managing public health disasters one of the first question is to ensure that the first responders are attended to. So, the front-line health care workers, the public health care managers of the disease for example the Drivers who drives these ambulances and nonmedical persons administrators, security organs who are directly involved in the response to the pandemic those should be prioritised. Then number two, the most vulnerable those who would otherwise die if they were not prioritised and these are the older persons and the persons with co-morbidities they should be prioritised. Then because COVID affects movement and social societal relationships that impacts on the economy then those parameters that are crucial for the progression of the society like school, places of worship. Then drivers of the economy like the tourism those should be prioritised. Because it is useless for you to be alive when you are poor poverty it's self it is a disease. IDI 010 male// bioethics/clinician

"Right now, the conversation on prioritization is not the right one. These vaccines were given to target healthcare and essential workers. Health care workers were prioritized in particular because they are at increased risk of infection. So far we have 1800 cases of health workers who have been infected and so far, the case fatality rate of health care workers is higher than that of the average citizen at 0.9."IDI 018 male//policy

"Vaccines are limited but let us immunize and vaccinate those who need it first. The people who are the front liners. We need to protect our front liners before we think of any other person it will help us. IDI 014 male//public

Whereas the dominant view was that health workers deserve priority, to some respondents the lay/ordinary citizens should access the vaccine before health workers because of the unequal risk of infection between the two groups. The reason for this was that by virtue of their wide knowledge on how to prevent infection and access to protective equipment, health workers are at a lower risk of infection than ordinary lay people who are largely ignorant. Others felt that individuals who frequently come into contact with many people should also get priority. For example, those who go work in crowded markets and find it hard to know who is sick and who is not.

"Sincerely sure and there are people who argue that doctors and health care workers should really get last because one they are equipped with all the knowledge of how to prevent it, yet the other population may not know how to prevent it. Two these health workers also have the resources to prevent the disease compared to the general public. So, I don't know what do you think about that?" IDI 012 male//scientist 
"Teachers also because they have to protect our teachers and then to protect our children and the rest. Then those who are above 50 when you are getting old, you have complications diabetes and the rest they start coming up. So, we also need to protect them so that we don't run out of old people on earth." IDI $014 \mathrm{male} / / \mathrm{public}$

"Identification of people, who are linking up with many people wherever they are just like they said that the health workers are the ones on the frontline yet for us we see that you are far away but look at the people in the markets, they interact with many people on a daily, the artistes move to various places day and night, imagine the people they meet and even infect. [...]. The taxi people also, they keep picking and dropping passengers at various destinations which increases the risk of spreading the infection. IDI 013 female//public

"It is populations at risk based on epidemiological analysis and it could be persons ages 60 and above, people with co-morbidities the diabetics, the hypertensives, the cancer patients, HIV maybe AIDs. So those are based on the final population which fits in that initial 20\%." IDI 001 female//policy

In literature about priority setting for access to scarce resources, there has been a debate about what has been described as the 'fair-innings argument' [33-37] and 'instrumental considerations' [38-40]. Part of this argument is that the young, who have not yet had a chance to live and experience life, should get priority because the elderly have already had a chance to live and the youth are more economically productive. Some reasoned that the elderly spend most of their time at home and have limited movements, yet they are the ones who are getting the vaccine first, while denying it to the young and active population that moves to several places, something they thought was wrong and would be difficult to accept.

"Implications of this is that the government tells us that the vaccine doses are not enough for all of us, now if you are to compare the life expectancy between the youth and the elderly in regard to who you would give the vaccine first, it should be the youth because they are on the edge of their life span, so we should give the vaccine to the young that shall have the energy to work. [...] Meet a group of young people and ask them who to give the vaccine between them and the elderly, you will be shocked with the responses, they will just tell you that we let the elderly die because they have had some time and enjoyed the world so we should give that same opportunity to the youth to enjoy as well [...]. IDI 013 female//public

In this study the criterion of 'vulnerability' stood out high as one of the factors that should determine who should be given priority for COVID-19 vaccination. However, there were varying views on what constitutes vulnerability, all of which are potentially acceptable. To some, vulnerability was understood in terms of compromised immunity and the risk of severe disease, others in terms of risk of being infected while others both of these. Considering this criterion, some suggested that health workers should get priority on account of the comparatively higher risk of infection by virtue their occupations, while those who recommended priority to the elderly was on the basis of their comparatively low immunity and risk of 
severe disease. However, considering the risk of being infected per se ran a risk of including on priority list, all those categories people whose occupations posed a higher risk of infection.

"Yeah, I am saying that the vaccine should even as a priority be given to the elderly because according to the discussions that we have heard or followed they are the most vulnerable and now the next ones would be the medical workers and that's what they are saying. [...] Two we are looking at those that are likely to be more vulnerable and may be that choice is dependent on that health workers, teachers who by their profession interface with the children may require that. I even heard that nursery school teachers are also saying that you come and also vaccinate us that we open our nurseries." IDI 006 male//lawyer

"What you have to understand about the whole situation my sister is we look at these things critically you know those that are prone to these issues these conditions created by COVID 19 are the old people. You see the problem we have is that the young people feel that they need to live but we need the wisdom of the old. [...] the issue is that the causalities we have with the young is limited unlike the old people. So, the old people should be given their issues, so we cannot say let us now wipe them out and neglect them because we need their wisdom, if we say we don't want them now we want the young people to survive and the rest and the rest then what happens to the old people? We need them also. [...] unless they young people who have problems already. You can be young, and you are old already you have heart issues, diabetes then we can now consider you. [...]." IDI 014 female// public

"[...] when you talk in terms of equity, everybody will want to access the vaccine at the same pace, but when you look at other factors like [...] you know [...] do we all have the same risks? Absolutely not, some people have a higher risk than others, for example the elderly 70 and above [...] ideally if the vaccines were enough, we could even have put it 62 or even 60 , so the elderly have a higher risk compared to others, even though they may not be very mobile in getting to these high transmission settings, but by virtue of their immune system and age, they are at higher risk." IDI 012 male//scientist

Some of the respondents' views were influenced by their perceptions of the looming vaccine hesitancy. Such respondents assigned priority in a manner that would mitigate this challenge. For this reason some respondents were of the view that individuals who can be regarded as influencers should be vaccinated first as strategy to ensure optimal uptake of the vaccine

"I think if you are trying to overcome these kinds of negative attitudes, you can find it beneficial to start off with opinion leaders who are respected and are likely to be a good influence [...] if you see them taking the vaccine, he would not advocate for something that could harm him, so it might be good for us [...] so I think [...] in a way you select who gets it first, and this is going to influence others, and I think it was very disheartening when the President said that he is still considering which one to use [...] because it means that with all the information that he has at his disposal and sitting with him right there [...] yes, the best information he has is that the vaccine is that the vaccine that is within the country is not the best [...] so, why would another person take it? Or why would another people advise another person to take it? I think it was a very unfortunate remark and I think actually there is no way you can counter that. [...] I think it was a well-considered opinion, it was not a random." IDI 011 male//scientist 
On the other hand, some respondents stressed the importance of considering other factors, beyond those considered by the $\mathrm{WHO}$ and guidance and consequently the local Vaccine deployment plan, which can make people vulnerable to both COVID-19 infection and severe disease outcomes. Some of such consideration that were cited include poverty, overweight/underweight, nutritional status, among others.

"Yes, I can even tell you that right now when you come to Mulago [National Referral Hospital] they will tell you we have five patients but you will find that four of them there's something to do we recent travel why they have COVID. If you remember at the beginning of the pandemic when we had few patients they were all related to recent travel. So I think people involved in the travel industry should be heavily prioritized." IDI 010 male//clinician/bioethicist

"[...] Personally, I believe that to be able to do a very good priority setting, you need very good data of who is in need where, who have we reached and who are we leaving behind? Because one of the most critical issues is that they should be able to reach the people that have the greatest need. So, we need to understand the epidemiological profile. Who is being affected? What is the burden of disease so that we know who it is that is driving infections and therefore who we must address based on that? That should be coupled with who are we able to reach? [...]" IDI 005 female//public health

"I am not sure but there is evidence that you had better prevent those that are more susceptible than those that are less, and this is scientific [...]." IDI 012 male//scientist

Respondents highlighted the need for contextualization of guidelines to the local setting if such guidelines are to be fit for purpose.

"The principle to me is the epidemiology when you are looking at the economics and social issues. [...] There are people out there if they don't work for one or two days they will die. You and me will survive, so there is vulnerability in itself in many ways vulnerability to infections, vulnerability socio economically and so many issues we need to think about so that we don't leave the poorest and most deprived people behind. And those people are also at highest risk of getting COVID because of the way they live. [...], keeping distance is not a privilege they have, and they also want to see their children go to school and some of them live across generations with the mother and grandmother and whatever and all the mix is there in the house. IDI 005 female//public health

On the other hand, some people reasoned that given the uncertainty about the risk profiles of the various vaccines being used, it was difficult to decide who should the vaccine first, just in case it was unsafe. For this reason some people recommended that those people who are important to society should not be vaccinated until the risk profiles of the vaccines have been fully established.

"In fact, the president should be the last because if you lose him then you're in trouble, I'm wondering if what we are reading on is correct like in case Magufuli dies what will happen, it will be very costly to the country, the president should be the last. [...] when they start with the medical workers we are in trouble 
because we are going to lose our very important resource that tells you how controversial your question is" IDI 006 male/lawyer/civil society

\section{The need for stakeholder engagement in priority setting}

On the issue of the process of priority setting for access to COVID-19 vaccine, the findings were also both descriptive and normative. Given that by the time of data collection the draft vaccine deployment plan had already been developed and accessed by some of the respondents, their responses were in the form of a descriptionof the status quo as opposed to what ought to have happened. However, at the same time, some of the responses went beyond describing the process as it had been and was going, to how it ought to be. For those who gave a descriptive account of the process of setting priorities for access to COVID-19 indicated that the process was more of a top-down approach, in which guidance adopted from the WHO and the COVAX facility.

Regarding the question on which stakeholders were involved in the process of setting priorities, the following explanations were given:

"First, we consulted with groups that were following the developments and had all the information available to us. We then converted our consultative group which comprised the Ministry of Health, academia, development partners, civil society and other government ministries, into a technical planning committee. [...] We have about three professors on that committee. We have people from the private sector, people from the media, civil society as well as the legal fraternity. We also have representatives from foreign missions and the UN. [...]. Everything else you see is in adaptation to that guidance, which is in addition to the global guidance that was provided by the WHO allocation framework. Our Vaccine Deployment Plan was appraised by the COVAX facility and found to be fit [...]." IDI0018 male//policy

Another respondent indicated that:

"Stakeholders have come from national, international, regional and of course the local hospital. International we have the WHO that keeps on giving guidelines on how to manage, recommending which vaccines to use etc. National level we have the presidential, commission then ministry of health. Some on the ministry of health are on the presidential taskforce and others. At level of ministry of health, they take care of the entire country because others are decision makers." IDI 012 male//scientist

On the other hand, those who provided a normative account of the process indicated a need for a bottomup approach, in which rigorous stakeholder engagement with more specific emphasis on community engagement should be a sine qua non for this process. Data obtained on this aspect indicates in high regard for scientific competence in equal measure as familiarity with local social and economic realities within the population. For this reason, on top of recommending that professional health workers who better understand the nature of the current disease, including its aetiology and mode of spread, should provide guidance in the process of priority setting, there was also concern that priorities were set without consulting stakeholders within communities and other population groups. The reasons for consultations 
with lay community members had to do with the fact that the locals know better who is at risk within their communities, and therefore, were better placed to advise on who ought to get priority for vaccination against COVID-19.

Apart from mentioning the stakeholders who are usually involved in the process of priority setting for access to health resources in general, some respondents suggested a number of categories of stakeholders they felt ought to be or should have been consulted and involved in the process of setting priority for access to COVID-19 vaccines. Apart from government, among the stakeholders that were highly recommended are lay members of the communities.

"I guess it should be the Ministry of Health should be in the fore front but we as also community it is important to guide whoever is going to vaccinate to have an input. because these communities have some uniqueness because maybe one time where you see the LC1 will be useful the time you may see the traditional chief might be more useful [...] " IDI 008 male//scientist

"Setting priorities especially for who can access the vaccine. The local council system is actually very effective. I have learnt that I think NRM's big achievement is creating that system of governance from local council level to the village, to the parish, to the system and local council people do know their residents very well. They know who is old, who is young, who is what age and all of that. It will be good if LCs are involved in identifying who is more susceptible to COVID in their community of course with a little education on why those kinds of particular people should be chosen. They can identify those people and present them but also in prevention mechanism because vaccination is a prevention mechanism." IDI 007 female//layer/civil society

"I believe in consultations a lot and I also believe in primary health care approaches because these vaccines ultimately are going back to the communities so that level of engagement of the structures that have been put up at the community level as representing society would have been very vital. The health management committees I still value them and I think if they were engaged sufficiently and given a lead within the districts they would have played a major role. District leadership would have defined it's self beyond the political set up to the technical people within the districts it would be very useful. Of course the national level at the ministry they would continue with their policy level but depending on the evidence they would have brought up. IDI 017 male//lawyer /civil society

Some respondent felt that the approached employed for this covid-19 vaccine priority setting fell short of what would have been desired by a number of stakeholders as:

"What I noted was there was a lot of paternalism. There was a lot of top-down approach in sense one will call it dictation by the people up labouring it down to the implementers. For example, in this hospital the directors of the hospital was not involved in the decision making on how his hospital space should be used it came from up. The minister also could have been disempowered because the decisions came from the national task force which was led by the prime minister. In making these decisions I can see that they were no meetings that are aware of, there were no consultative meetings, no discussions, no think- 
tanks whether public or private and people just decided paternalistically this is what is supposed to be done." IDI 010 male//clinician/bioethicist

"Well, maybe there could have been a more systematic way of first of all, getting views and that could have happened, and again it is not my field of expertise so maybe they sought those views from those that are specialized in that area, and once they have those views, then they come up with guidelines." IDI 011 male//scientist

To emphasize the need to involve communities in priority setting for access to COVID-19 vaccine, some respondents indicated that one the mistakes on the part of government had been failure to involve communities in the process. One justification for community involvement was that the involvement of the community in the process creates goodwill in implementing the program.

"The second challenge is the lack of involvement of the community and their consultation. People are always feel left out and they are not part of this because of that they will not join your efforts yet you need community goodwill for any successful public health campaign." IDI 010 male//clinician/bioethicist

"To a successful program so the Government through this ministry of health and other sectors should get an adequate preparation to involve the people down. For example if 100 vaccines were delivered at the village level and the village were asked to prioritise who is going to receive? Imagine the village dynamics would they give it to the 30 year older persons in that village first? Would they prioritise it for the teachers in that village? That kind of grass root involvement is what I think should come in as the main factor. Then the other one is diminishing the paternalism and dictatorship by the government and giving the technical persons, because I can assure you technical persons if they were given adequate time to have in the input in the COVID response and particularly the issue of the vaccine response they have not." IDI 010 male/clinician/bioethicist

In the opinion of some of the respondents, one of the activities that should have been conducted as part of community engagement is community education and sensitization about vaccination, including explanations and justifications of the priorities.

"Educating people, educating communities, doing that awareness. [...]. So, education and letting people know. Coz if we see how the vaccine looks maybe on TV, in these visual materials they are using on the billboard how it is packaged, who can move with the vaccine, who is supposed to give the vaccine? Where is the vaccine given? Yeah remember the days of kick polio out of Uganda. We knew who gives the polio vaccine, we knew where the polio vaccine is taken. We knew who can talk about polio. IDI 007 female//lawyer/civil society

"Priorities were not set basing on any condition, may be the Ministry just thought that these are the priorities because they didn't do a questionnaire to see that these are the priorities because you can't say that it's only the teachers that are a priority. For example, if you look at the young children who are coming from my home who go to school and come back, who is more exposed to danger, me the mother 
of that child, my husband plus the siblings. So I feel that the people they felt that they are more risky they are not, we are at more risk because for them they have the information and they are few, when you look at the family or the community where we at, that's why they said don't go for burial but if I lose someone like my sister or brother will I stay home, definitely I can't. So, in terms of selection basically me I feel that they did it without consideration, they just made the decision themselves without doing the consultation because maybe there was no time to do that small survey so that we know who is priority? IDI 013 female//public

To emphasize the need for stakeholder involvement in the process of priority setting for access to COVID19 vaccine, some respondents warned about the possibility of the public disagreeing with the resulting list of priorities if they are neither involved in the process, nor given a justification as why they miss on the priority list.

"So, what you want to tell us will it only imply that only those that will be vaccinated are the ones to perform or work, what will happen to the rest of us, we won't agree because we want to have an answer that if I'm one of those that have not got the vaccine, what should I do because I have to survive. ... They won't be willing to wait, why? Because they have waited for a full year, there's no person that you will again tell to stay home when the lock down is lifted. IDI 013 female//public

"Again, every time you deal with priority, there are issues of class, decision making, so generally they revolve around those two so there will always be some controversies. You see once you say that you are priority then I have to ask myself on what basis and that definitely brings in that controversy inherently. IDI 006 male//lawyer/civil society

"It has a lot to do with who makes the decision now, controversy would arise as of who makes the decision, would that decision have to be made by parliament, executive or the health fraternity, who makes the decision, controversy would always be there in regard to that. Secondly, what is the consideration, is it social or political, age or social status, so the controversy would evolve around that, the characterization of priorities, so that's where I see controversy." IDI 006 male//lawyer/civil society

\section{Discussion}

The study set out to assess stakeholders' perspectives on priority setting for access to COVID-19 vaccine as part of evidence that should guide worthwhile priority setting for vaccine access and distribution among Ugandans, as well as ignite public debate on this subject. Our findings show that there are significant although hush controversies about the status quo of the country's priorities for access to COVID-19 vaccines. The controversies identified in this study, specifically those that arose from the variables on which this paper has reported include, insufficient clarity about the existence of elaborate official guidelines on how to ration COVID-19 vaccines; the evidence that was used to set such priorities and the process that was followed to that effect. 
Currently, national guidelines for vaccination against COVID-19 are in a draft form while priority setting for the guidelines is being informed by the general WHO and COVAX guidelines to the letter as no effort was made to contextualize them to suit local epidemiological, demographic, social, economic and other realities. Whereas findings largely support priorities for vaccination as prescribed by the WHO and the COVAX facility, views identified in this study strongly suggest that this guidance should have been localized to ensure that it is responsive to the local dynamics of the population. In our opinion, this is important for at least three reasons: one, the social dynamics between countries differ greatly in ways relevant to the goals of COVID-19 vaccination. For example, Uganda has a very low rate of urbanization is less than a quarter of the total population, and majority of the elderly populations live in the countryside [41] where physical distancing is very possible. Following from this fact, it could be argued that the urban population who are more exposed to a high risk of infection by dint of the nature of their work and environment generally, be prioritized before rural populations. Secondly, in Uganda, most of the infections were related or had a history of travel and their associated contacts and the peak of the first wave, most of the community infection was associated with long distance cross boarder truck driver who continued to move throughout east Africa even at the time of lockdown. These would be prioritised because they acted as the major movers of the disease. And thirdly, whereas the WHO and Covax facility guidelines were evidence-based and clear about which categories of people should get priority for COVID-19 vaccination, the extreme scarcity of the vaccines in Uganda meant that it was not possible to cover all those groups that were listed as priority. Hence, there should have a local process of setting priorities among the priorities recommended by the $\mathrm{WHO}$ and the Covax facility.

Another major finding of this study is that many people in the general public, including the elite, are unaware of the existence of guidelines for decisions of who gets the vaccine first and who waits. Among those who have heard about them, they do not know much about the content of the guidelines apart from what they hear in passing during some of the media briefings by the Ministry of health officials. Despite this lack of knowledge of the guidelines, there was wide agreement on the categories of people who should be given priority and reasons it. Majority were of the view that health workers and the elderly and generally those with compromised immunity should get first priority. However, a significant number of our respondents deviated from the general thinking about who gets priority and why. This deviation can be accounted for by their seeming conception of vulnerability and risk, specifically in the context of COVID19 vaccination. To these people, the concept of vulnerability should not simply be defined in terms of compromised immunity, but also in terms or degree of risk of infection due to people's lifestyles as a risk factor for infection, more especially the nature of work. Consideration of this factor would, however, that priority should extend from health workers to other groups such as, people in the transport sector, business (especially congested markets), urban dwellers, travellers and groups whose lifestyles makes physical distancing less feasible.

But equally interesting was how some people think about risks of COVID-19 infection. For example, whereas it is generally taken for granted the health workers are comparatively at higher risk of infection, some respondents reasoned that by virtue of their knowledge (about transmission) and facilities such as personal protective equipment, health workers had less risks of infection compared to the majority of the 
population who lack such knowledge and facilities. Whereas this kind of reasoning may be weak, it is not entirely irrational, and has important implications. The implication is that unless communities are engaged rigorously to enable them understand the reasoning behind decisions that affect them, there are chances of seeing such decisions as unjustified, hence reduce their good will and support of the program.

There was yet another noteworthy dimension of risk perception, specifically with regard to COVID-19 vaccination. Whereas the popular assumption was that delay to access the vaccine was a risk to be avoided, instead to some people 'risk' was in the form of uncertainty about safety profile of COVID-19 vaccine. To such people, it would be unwise to 'experiment' on health workers and other highly valuable individual and groups within society. To such people, therefore, those who are not very useful to society should have received the vaccine first, while those who are considered most useful to society should be vaccinated last. It is important to notice that such views can be big drivers of vaccine hesitancy. Hence, since effectiveness is one of the major goals of the COVID-19 vaccination program, then rigorous and continuous community engagement is necessary as a strategy to identify and address such conceptions.

Further, it was noticed generally about the issue of who gets priority and reasons that, is that most views implied more of pragmatic (effectiveness) considerations and less of equity considerations. That is, the overarching consideration in deciding who should be vaccinated first is reducing the risk of widespread infection (target not just to the elderly, but those with risky lifestyles), and protect the health system (healthcare workers). However, instrumental considerations beyond immediate health workers has always been a controversial criterion in the debates about the allocation of scarce health resources (....)

\section{Conclusion}

In order to ensure effectiveness and equity in the design and implementation of programs such as vaccination against COVID-19, there can be no substitute for rigorous community engagement. It also emerged that whereas it is important to follow standardized guidance of how to roll out programs, the findings of this study indicate that in order to ensure effectiveness of such guidance there is need to retailor such guidance to different local contexts. Further, whereas theoretical positions on who should get priority in accessing the available scarce health interventions need to balance efficiency and equity, this study has identified bias in favour of effectiveness-cum-efficiency in the way the few vaccines are rationed. With regard to effectiveness, people seemed to be more concerned more about achieve the goal of reducing the rates of infection transmission of the disease as opposed to health outcomes once a person has been infected.

\section{Declarations}

Ethics approval and consent to participate: Ethical review and approval was appropriately sought from the Makerere University School of Health Sciences Research and Ethics Committee SHSREC: 2020-043, followed by clearance by the Uganda National Council for Science and Technology HS1101ES. Only adult individuals of 18 years and above both males and females who provided written informed consent 
participated in the study. No participant identifying information was recorded. All the methods were carried out in accordance with relevant national and international guidelines and regulations.

Consent for publication: Not Applicable.

Availability of data and materials: The datasets used and/or analysed during the current study are available from the corresponding author on reasonable request.

Competing interests: All authors have no conflict of interest to declare.

Funding: Research reported in this publication was supported by the WHO: HEG COVID-19 Sep 2020. The content is solely the responsibility of the authors and does not necessarily represent the official views of the National Institutes of Health.

Authors' contributions: JO conceptualized the study. All authors participated in data collection and analysis. After this state all authors agreed on the themes that should reported in this manuscript. JB developed the daft manuscript and shared it with $\mathrm{JO}$ and BK who provided suggestions on how to improve the write-up. All authors approved the manuscript.

Acknowledgements: We a grateful to all the respondents who participated in the study.

\section{References}

1. Weekly epidemiological update on COVID-19 - 31 August 2021

[https://www.who.int/publications/m/item/weekly-epidemiological-update-on-covid-19--31-august2021]

2. WHO African Region numbers at a glance [https://www.afro.who.int/health-topics/coronavirus-covid19]

3. Shen AK, Hughes Iv R, DeWald E, Rosenbaum S, Pisani A, Orenstein W: Ensuring Equitable Access To COVID-19 Vaccines In The US: Current System Challenges And Opportunities: Analysis examines ensuring equitable access to COVID-19 vaccines. Health Affairs 2021, 40(1):62-69.

4. McClung N, Chamberland M, Kinlaw K, Matthew DB, Wallace M, Bell BP, Lee GM, Talbot HK, Romero JR, Oliver SE: The Advisory Committee on Immunization Practices' ethical principles for allocating initial supplies of COVID-19 vaccine-United States, 2020. Morbidity and mortality weekly report 2020, 69(47):1782.

5. Bollyky TJ, Gostin LO, Hamburg MA: The equitable distribution of COVID-19 therapeutics and vaccines. Jama 2020, 323(24):2462-2463.

6. Wouters OJ, Shadlen KC, Salcher-Konrad M, Pollard AJ, Larson HJ, Teerawattananon Y, Jit M: Challenges in ensuring global access to COVID-19 vaccines: production, affordability, allocation, and deployment. The Lancet 2021. 
7. Herzog LM, Norheim OF, Emanuel EJ, McCoy MS: Covax must go beyond proportional allocation of covid vaccines to ensure fair and equitable access. bmj 2021, 372.

8. Herlitz A, Lederman Z, Miller J, Fleurbaey M, Venkatapuram S, Atuire C, Eckenwiler L, Hassoun N: Just allocation of COVID-19 vaccines. In.: BMJ Specialist Journals, 2021.

9. National Academies of Sciences E, Medicine: Framework for equitable allocation of COVID-19 vaccine. 2020.

10. Torjesen I: Covid-19: Pre-purchasing vaccine-sensible or selfish? In.: British Medical Journal Publishing Group, 2020.

11. Bollyky TJ, Bown CP: The tragedy of vaccine nationalism: only cooperation can end the pandemic. Foreign Aff 2020, 99:96.

12. Aborode AT, Olofinsao OA, Osmond E, Batubo AP, Fayemiro O, Sherifdeen O, Muraina L, Obadawo B, Ahmad S, Fajemisin EA: Equal Access of COVID-19 Vaccine distribution in Africa: Challenges and Way Forward. Journal of Medical Virology 2021.

13. Bibbins-Domingo $K$, Petersen M, Havlir D: Taking vaccine to where the virus is-equity and effectiveness in coronavirus vaccinations. In: JAMA Health Forum: 2021: American Medical Association, 2021: e210213-e210213.

14. Jean-Jacques $M$, Bauchner $H$ : Vaccine distribution-equity left behind? JAMA 2021, 325(9):829-830.

15. Press VG, Huisingh-Scheetz M, Arora VM: Inequities in technology contribute to disparities in COVID19 vaccine distribution. In: JAMA Health Forum: 2021: American Medical Association, 2021: e210264-e210264.

16. Nhamo G, Chikodzi D, Kunene HP, Mashula N: COVID-19 vaccines and treatments nationalism: Challenges for low-income countries and the attainment of the SDGs. Global public health 2021, 16(3):319-339.

17. Hassoun N: Against vaccine nationalism. Journal of Medical Ethics 2021.

18. Jha P, Jamison DT, Watkins DA, Bell J: A global compact to counter vaccine nationalism. The Lancet 2021, 397(10289):2046-2047.

19. Chohan UW: Coronavirus \& Vaccine Nationalism. 2021.

20. GoU: Uganda receives 864,000 doses of COVID-19 vaccines. In., 2021.

21. Africanews: Uganda struggles to vaccinate against Covid amid fears of a new wave. In., 2021.

22. WHO: An allocation framework for fair and equitable access to COVID-19 health products 2020.

23. Anand S, Hanson K: DALYs: efficiency versus equity. World Development 1998, 26(2):307-310.

24. Diderichsen F: Resource allocation for health equity: issues and methods. 2004.

25. Jehu-Appiah C, Baltussen R, Acquah C, Aikins M, d'Almeida SA, Bosu WK, Koolman X, Lauer J, Osei D, Adjei S: Balancing equity and efficiency in health priorities in Ghana: the use of multicriteria decision analysis. Value in health 2008, 11(7):1081-1087.

26. Culyer A: Efficiency, equity and equality in health and healthcare. F1000Research 2019, 8(800):800. 
27. Vågerö D: Equity and efficiency in health reform. A European view. Social science \& medicine 1994, 39(9):1203-1210.

28. Lane $\mathrm{H}$, Sarkies $M$, Martin J, Haines $\mathrm{T}$ : Equity in healthcare resource allocation decision making: a systematic review. Social science \& medicine 2017, 175:11-27.

29. Wagstaff A: QALYs and the equity-efficiency trade-off. Journal of health economics 1991, 10(1):2141.

30. Cuadras-Morató X, Pinto-Prades JL, Abellán-Perpiñán JM: Equity considerations in health care: the relevance of claims. Health Economics 2001, 10(3):187-205.

31. Bebbington A, Davies B: Equity and efficiency in the allocation of the personal social services. Journal of Social Policy 1983, 12(3):309-329.

32. Tao Y, Henry K, Zou Q, Zhong X: Methods for measuring horizontal equity in health resource allocation: a comparative study. Health economics review 2014, 4(1):1-10.

33. Nord E: Concerns for the worse off: fair innings versus severity. Social science \& medicine 2005 , 60(2):257-263.

34. Rivlin MM: Why the fair innings argument is not persuasive. BMC Medical Ethics 2000, 1(1):1-6.

35. Williams A: Intergenerational equity: an exploration of the 'fair innings' argument. Health economics 1997, 6(2):117-132.

36. Bognar G: Fair innings. Bioethics 2015, 29(4):251-261.

37. Williams M: Rationing health care. Can a" fair innings" ever be fair? BMJ: British Medical Journal 1997, 314(7079):514.

38. Brock DW: Separate spheres and indirect benefits. Cost effectiveness and resource allocation 2003, 1(1):1-12.

39. Rawlings A, Brandt L, Ferreres A, Asbun H, Shadduck P: Ethical considerations for allocation of scarce resources and alterations in surgical care during a pandemic. Surgical endoscopy 2021, 35(5):2217-2222.

40. Persad G, Wertheimer A, Emanuel EJ: Principles for allocation of scarce medical interventions. The Lancet 2009, 373(9661):423-431.

41. UBOS: Statistical Abstract. In., 2020. 